\title{
Structural Design of Self-stressed Roof Structures using Vector-based Graphic Statics and Numerical Optimization
}

\author{
P. D'Acunto*, P.O. Ohlbrock ${ }^{\dagger}$ and T. Oberbichler ${ }^{\dagger 1}$ \\ * Professorship of Technology Design, Department of Architecture \\ Technical University of Munich (TUM) \\ Arcisstraße 21, 80333 Munich, Germany \\ e-mail: pierluigi.dacunto@tum.de \\ ${ }^{\dagger}$ Chair of Structural Design, Department of Architecture \\ Swiss Federal Institute of Technology in Zurich (ETH Zurich) \\ Stefano-Franscini-Platz 1, 8093 Zurich, Switzerland \\ e-mail: ohlbrock@arch.ethz.ch \\ ${ }^{\dagger 1}$ Chair of Structural Analysis, Department of Civil, Geo and Environmental Engineering \\ Technical University of Munich (TUM) \\ Arcisstraße 21, 80333 Munich, Germany \\ e-mail: thomas.oberbichler@tum.de
}

\begin{abstract}
The ability to work with both form and forces is of particular relevance to the structural design process, especially in the conceptual design phase, as these are among the most important parameters that affect the static behaviour of a structure. In this respect, graphic statics is a powerful geometric framework for the conceptual design of structures in static equilibrium. Thanks to the interdependency between form and force diagrams in graphic statics, it is generally possible to modify one of the diagrams and visually evaluate the resulting transformation of the other diagram. A recent development to extend graphical statics to the third dimension has introduced a generalized procedure for the construction and transformation of vector-based 3D force diagrams, for any 3D form diagram of a pin-jointed structure in static equilibrium [1]. This approach, coupled with equilibrium-based form-finding methods, such as the Combinatorial Equilibrium Modelling [2], allows for quick and interactive exploration of possible equilibrium solutions during the conceptual design process. However, when constraints are introduced into the design problem, the initial form-finding procedure used for the construction of form and force diagrams, and the subsequent mutual transformation of these diagrams define nonlinear problems that cannot be solved directly. In this case, numerical optimization approaches can be used to approximate the design solution. In this contribution, an interactive workflow is presented for the structural design of self-stressed roof structures that combines graphical methods and numerical optimization. Various geometric constraints can be applied to both form and force diagrams during the initial form-finding and the subsequent transformation of the diagrams. As shown by the presented case studies, these constraints can take into account specific boundary conditions or material and fabrication requirements, which can thus become active parameters of the structural design process.
\end{abstract}
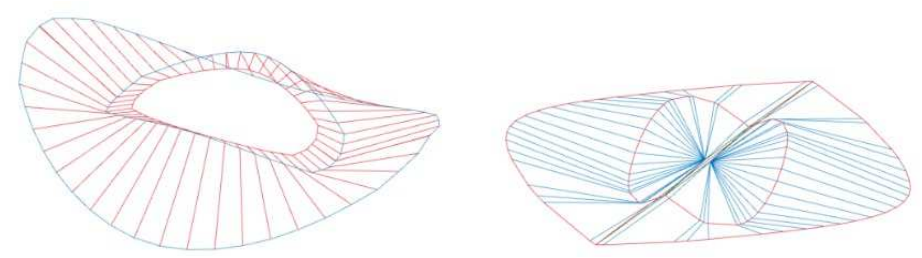

Fig.1 (left) Form diagram of a self-stressed structure; (right) corresponding force diagram

\section{REFERENCES}

[1] P. D'Acunto, J.-P. Jasienski, P.O. Ohlbrock, C. Fivet, J. Schwartz and D. Zastavni, "Vector- based 3D graphic statics", International Journal of Solids and Structures, 167, 58- 70 (2019).

[2] P.O. Ohlbrock and P. D'Acunto, "A Computer-aided Approach to Equilibrium Design based on Graphic Statics and Combinatorial Variations", Computer-Aided Design, 121, 102802 (2020). 